\title{
Panuveitis with disc edema after dengue fever: A rare presentation
}

\section{Radha Annamalai,} Kalpana Suresh

Department of Ophthalmology, Sri Ramachandra University, Porur,

Chennai, Tamil Nadu, India

Address for the Correspondence:

Dr. Radha Annamalai, Sri Ramachandra University, Porur, Chennai - 600 166,

Tamil Nadu, India.

E-mail: drradhaannamalai@ yahoo.co.in

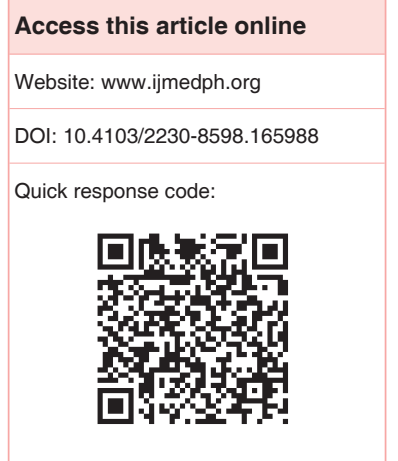

We report a case of a 47 year old male who presented with panuveitis and disc oedema two weeks after an episode of dengue fever. He had complaints of headache with pain and defective vision in his right eye that had developed during his recovery from dengue fever. We treated him with topical steroid drops and subtenon steroid injections along with atropine eye drops. Oral prednisolone was started after 1 week on his review visit following which his symptoms resolved and vision improved. This case is being presented as panuveitis with disc oedema is a rare complication of dengue fever and also because the patient responded well to medical management with restoration of his vision.

Key words: Dengue fever, disc edema, macular edema, panuveitis, steroid

\section{INTRODUCTION}

Panuveitis is known to be a progressive inflammation that can cause blinding complications. It is a generalized inflammation of not only the whole of the uveal tract, but also involves the retina and vitreous humor. Etiology can be infectious or noninfectious. Tuberculosis and Vogt-Koyanagi-Harada syndrome are the most common causes of panuveitis in India. Dengue fever (DF) is a mosquito-borne disease that is commonly found in the tropics and is known to be associated with ocular inflammation. The widening spectrum of ophthalmic complications in dengue infection suggests one to conclude that several pathophysiologic processes are involved. ${ }^{[1]}$

\section{CASE REPORT}

A 47-year-old male patient attended the outpatient department complaining of pain, redness, defective vision in the left eye with the headache of 1-week duration. A detailed history revealed that he had been diagnosed as DF for which he had been treated elsewhere. He was an alcoholic and had been diagnosed with cirrhosis liver for which he was undergoing treatment.

He was admitted with fever, headache and arthralgia in a local hospital, 2 weeks prior to the onset of blurring of vision. At that time, the investigations were as follows. Erythrocyte sedimentation rate $61 \mathrm{~mm}$ in $1 \mathrm{~h}$ (Westergren's method), hemoglobin: $12.5 \mathrm{~g} \%$, total count: 14000 cells/cubic mm. Differential count showed polymorphs $62 \%$, lymphocytes $28 \%$, eosinophils $8 \%$, and basophils $2 \%$. Platelet count was $1.0 \mathrm{lakh} /$ cubic mm. Random blood sugar was $180 \mathrm{mg}$. Purified protein derivative and chest X-ray were normal. Enzyme-linked immunosorbent assay (ELISA) test for HIV and tests for syphilis serology were negative.

The lesions in the eye and anterior segment were not suggestive of herpetic etiology, and we did not suspect zoster or simplex. Besides the patient did not consent for an anterior chamber tap and hence further investigations could not be performed. CD count was 700 cells per cubic mm and ELISA for HIV1 and 2 were negative. We treated this patient in concurrence with the physician who had examined him for joint and other rheumatic diseases, and he was not suffering from any joint disease. Dengue ELISA test was positive from the serum sample 3 days after the fever started. He was given symptomatic treatment, which included antipyretic and analgesic tablets and was discharged once the fever subsided. After 1-week he complained of defective vision of the right eye and was seen by us. On ophthalmic examination, his bestcorrected visual acuity was 6/60, N18 in the right eye and 6/6, N6 in the left eye. Slit-lamp examination of 
the right eye revealed fresh keratic precipitates in an inferior distribution. Anterior chamber showed cells $3+$ and flare grade 3 with fibrin and posterior synechiae [Figure 1]. The pupil was sluggish in reaction. Fundus examination revealed grade 2 vitreous cells, optic disc edema, intra-retinal hemorrhages, hard exudates and areas of retinitis with macular edema in the right eye [Figure 2]. Examination of the left eye was normal. Intraocular pressure by applanation tonometry was 16 $\mathrm{mmHg}$ in both the eyes. $1 \%$ atropine eye drops, $1 \%$ prednisolone eye drops and subtenon triamcinolone acetonide injection were administered to the right eye. Optical coherence tomography (OCT) showed macular edema in the right eye [Figure 3]. Examination of the left eye was normal. Fluorescein angiography was performed 3 days after starting treatment. It was characterized by leakage from vessels and blocked fluorescence due to retinal hemorrhage. The area of retinitis showed staining in the late phase of the angiogram. A diagnosis of panuveitis RE was made. Since there was no fever and presence of optic nerve head involvement, he was started on a course of systemic steroids. Tablet Prednisolone $1 \mathrm{mg} / \mathrm{kg}$ body weight was started and tapered over 6 weeks in concurrence with his diabetologist. Examination on review after 1-month showed that the best-corrected visual acuity was 6/36, N12 in right eye and 6/6, N6 in the left eye. Fundus examination revealed the total resolution of disc edema with a decrease in macular edema and the retinal hemorrhages. Color vision testing using Ishihara's pseudo isochromatic chart and visual field analysis by Humphrey field analyzer were normal.

\section{DISCUSSION}

Dengue is the most common mosquito-borne viral disease in humans. Both DF and dengue hemorrhagic fever (DHF) are known to be self-limiting with systemic sequelae. The early phase of DHF is indistinguishable from DF. DHF is defined by World Health Organization as DF associated with thrombocytopenia and hemoconcentration. Its most severe form, dengue shock syndrome, is associated with hypotension, narrowing of pulse pressure and circulatory failure. The diagnosis can be confirmed by detection of dengue-specific IgM antibodies (IgM enzyme-linked immunoassay).

Dengue virus infection has recently been reported to cause different ocular manifestations like subconjunctival hemorrhage, ${ }^{[2]}$ nongranulomatous anterior uveitis, focal chorioretinitis with or without macular edema, macular hemorrhages, ${ }^{[3]}$ retinal hemorrhages ${ }^{[4]}$ retinal vasculitis (periphlebitis), vitritis, intermediate uveitis and uncommonly, optic neuritis or papillitis. Bilateral vitreous hemorrhage, ${ }^{[5]}$ bilateral choroidal effusions ${ }^{[6]}$ and bilateral optic neuritis have been reported to occur after $\mathrm{DF}^{[7]}$ though less frequently.

The spectrum of ophthalmologic manifestations would lead one to conclude that several pathophysiologic processes are involved. The first and most obvious pathogenesis would be the thrombocytopenic state, with its resultant bleeding tendency, which gives rise to increased incidence of hemorrhage. These hemorrhages manifest as retinal blot hemorrhages in the macula and retinal periphery.

It has been suggested that the pathogenesis of these ocular manifestations are directly due to the immunopathogenesis of DF.

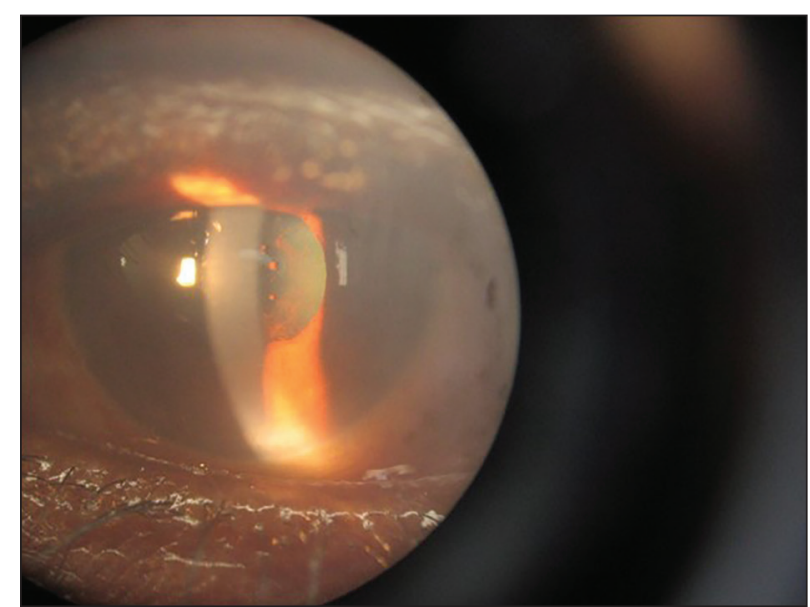

Figure 1: Anterior segment inflammation

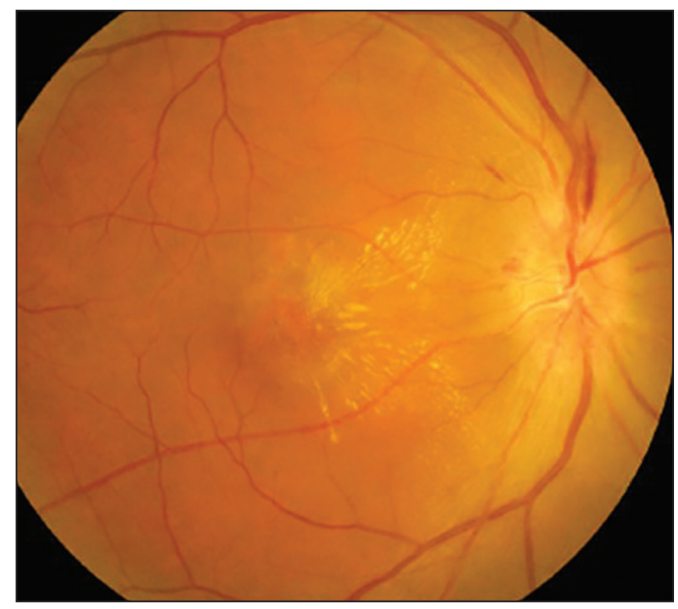

Figure 2: Fundus photograph of the right eye showing retinitis with disc edema

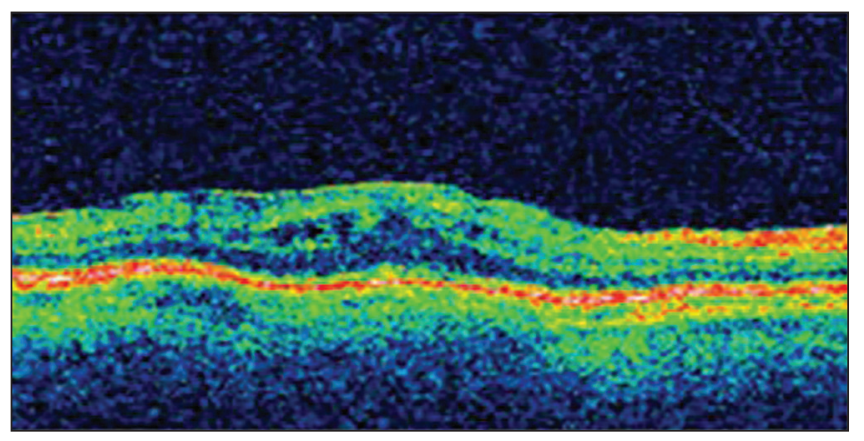

Figure 3: Optical coherence tomography of the right eye shows macular edema

Other postulates include viral mutations, viral virulence, and host susceptibility. Viral genetic mutations have been demonstrated to occur within the various serotypes; however, the biologic effects induced by these mutations are as yet uncharacterized.

Dengue fever resulting in a permanent visual loss in both eyes has been proposed to occur due to retinal capillary occlusion. ${ }^{[8]}$ However, clinical presentation and behavior of these complications suggest an 
immunogenic etiology rather than an infective one. Patients manifest complications in varying degrees of severity after a week of onset of their viral febrile illness. This is the time when most patients with the uncomplicated disease are just about to recover both systemically as well as hematologically and could correlate to the body's increasing immunologic response. Ocular complications associated with DF are rare but may result in permanent visual impairment..$^{[9]}$ Reports of spontaneous resolution of dengue induced ophthalmic inflammation exist. As there was panuveitis with optic disc involvement in our case we felt systemic steroids were indicated. Delayed onset of the eye problem after the fever subsided and prompt response to steroids in our case points towards an immune-mediated underlying cause. In our case, visual recovery had been very satisfactory probably because there was no delay in starting systemic steroids. Being a single case report, this result cannot be generalized to draw a conclusion regarding the prognosis of panuveitis in dengue. We may be able to get a better picture once larger case series are published. To conclude, dengue panuveitis, should be kept as a differential diagnosis of any panuveitis with neuroretinitis. Fundus fluorescein and indocyanine green angiography, OCT, and visual field testing are useful tools in the diagnosis of dengue maculopathy, which can cause severe visual loss. ${ }^{[10]}$ We feel that to understand better and manage the full spectrum of ocular disease caused by dengue infection, all patients who have suffered from DF should be referred for detailed ophthalmologic evaluation.

\section{REFERENCES}

1. Chan DP, Teoh SC, Tan CS, Nah GK, Rajagopalan R, Prabhakaragupta MK, et al. Eye institute dengue-related ophthalmic complications workgroup. Ophthalmic complications of dengue. Emerg Infect Dis 2006;12:285-9.

2. Kapoor HK, Bhai S, John M, Xavier J. Ocular manifestations of dengue fever in an East Indian epidemic. Can J Ophthalmol 2006;41:741-6.

3. Deutman AF, Bos PJ. Macular bleeding in dengue fever. Klin Monbl Augenheilkd 1979;175:429.

4. Chlebicki MP, Ang B, Barkham T, Laude A. Retinal hemorrhages in 4 patients with dengue fever. Emerg Infect Dis 2005;11:770-2.

5. Nainiwal S, Garg SP, Prakash G, Nainiwal N. Bilateral vitreous haemorrhage associated with dengue fever. Eye (Lond) 2005;19:1012-3.

6. Cruz-Villegas V, Berrocal AM, Davis JL. Bilateral choroidal effusions associated with dengue fever. Retina 2003;23:576-8.

7. Aragão RE, Barreira IM, Lima LN, Rabelo LP, Pereira FB. Bilateral optic neuritis after dengue viral infection: Case report. Arq Bras Oftalmol 2010;73:175-8.

8. Siqueira RC, Vitral NP, Campos WR, Oréfice F, de Moraes Figueiredo LT. Ocular manifestations in Dengue fever. Ocul Immunol Inflamm 2004:12:323-7.

9. Lim WK, Mathur R, Koh A, Yeoh R, Chee SP. Ocular manifestations of dengue fever. Ophthalmology 2004;111:2057-64.

10. Bacsal KE, Chee SP, Cheng CL, Flores JV. Dengue-associated maculopathy. Arch Ophthalmol 2007;125:501-10.

How to cite this article: Annamalai R, Suresh K. Panuveitis with disc edema after dengue fever: A rare presentation. Int J Med Public Health 2015;5:393-5.

Source of Support: Nil, Conflict of Interest: None declared. 